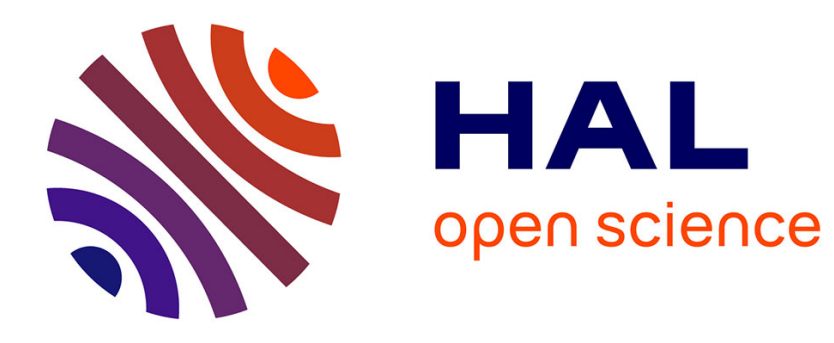

\title{
Evolution of Layered Structures in a Gradient-Dependent Viscoplastic Material
}

C. Faciu, A. Molinari

\section{To cite this version:}

C. Faciu, A. Molinari. Evolution of Layered Structures in a Gradient-Dependent Viscoplastic Material. Journal de Physique IV Proceedings, 1996, 06 (C1), pp.C1-45-C1-54. 10.1051/jp4:1996105 . jpa00254136

\section{HAL Id: jpa-00254136 https://hal.science/jpa-00254136}

Submitted on 1 Jan 1996

HAL is a multi-disciplinary open access archive for the deposit and dissemination of scientific research documents, whether they are published or not. The documents may come from teaching and research institutions in France or abroad, or from public or private research centers.
L'archive ouverte pluridisciplinaire HAL, est destinée au dépôt et à la diffusion de documents scientifiques de niveau recherche, publiés ou non, émanant des établissements d'enseignement et de recherche français ou étrangers, des laboratoires publics ou privés. 


\title{
Evolution of Layered Structures in a Gradient-Dependent Viscoplastic Material
}

\author{
C. Făciu and A. Molinari* \\ Institute of Mathematics of the Romanian Academy, P.O. Box 1-764, 70700 Bucharest, Romania \\ * Laboratoire de Physique et Mécanique des Matériaux, URA 1215 du CNRS, Université de Metz, Ile du \\ Saulcy, 57045 Metz cedex 1, France
}

\begin{abstract}
In this paper we discuss a new model to the problem of phase transformations. This model provides a modification of Maxwell's rate-type constitutive equation [1], by incorporating higher order straingradients in the relaxation function. Thermodynamical aspects and the compatibility with the unmodifed Clausius-Duhem inequality are discussed. Numerical results show that the model is able to describe the creation and propagation of regular patterns of martensitic and austenitic stripes during stress-induced phase transitions in shape memory single crystals.
\end{abstract}

\section{INTRODUCTION}

Phase transitions in continuum bodies are a subject of intense studies in mechanics, mathematics and metallurgy. Recently, it was shown (see [1] and the references given there) that a Maxwell's rate-type viscoelastic constitutive equation, with a non-monotone equilibrium stress-strain relation can predict specific phenomena accompanying stress-induced phase transitions and plastic Aow localization in metallic materials during uniaxial tension tests in a rigorous mathematical framework. On the other hand, gradient terms have been introduced repeatedly by physicists in phenomenological theories of phase transitions, in order to address various problems related to the instability and localization of deformation (see for instance $[2,3,4]$ ).

By combining these two different approaches in a common constitutive structure, we have proposed [5] a new model to the problem of phase transitions in solid bars with microstructures. The present approach incorporates three types of mechanism which compete to the description of nonequilibrium phase transitions. These are the "softening mechanism" due to the non-monotonicity of the homogeneous equilibrium curve, the "viscous mechanism" which is responsible of the interface kinetics, as for the Maxwellian model, but also an internal length scale ("strain gradient effects").

In the first part of this paper we give a short survey on local and non-local phenomenological theories of phase transitions. We outline the advantages and disadvantages of these approaches botti from mathematical and thermodynamical point of view. In this contex the new model is des.ribed and it is showed how the unmodified Clausius-Duhem inequality requires it to be a viscoplastic one.

In the last section we show by means of numerical examples how the presence of higher order strain gradients leads to the formation of an increasing number of interfaces into the specimen. These solutions of strain controlled initial-boundary value problems can be associated with layered structures as they appear in the process of nucleation and growth of martensitic zones insides austenitic phase.

\section{PHASE TRANSITIONS AS FIELDS THEORIES}

We consider in the following phenomenological theories of phase transitions as fields theories, i.e., they are described as solutions of initial-boundary value problems for the fields equations. All over this paper we discuss only the one-dimensional, isothermal case, as the essence of the arguments 
is best illustrated.

The particles of a one-dimensional body $\mathcal{B}$ are labelled by their position $X \in \mathcal{R}$ (Lagangian coordinate), where $\mathcal{R}$ is a finite or infinite interval on the real line, called the reference configuration. The uniform density of the body in the reference configuration is denoted by $\rho>0$. A one dimensional motion of the body $\mathcal{B}$ is a mapping $x=\chi(X, t), X \in \mathcal{R}, t \geq 0$, with the property that for any fixed $t, \chi(\cdot, t)$ is injective and bicontinuous with respect to $\mathrm{X}$. The number $x$ (Eulerian coordinate) is the position of the particle $X$ at time $t . \chi(\cdot, t)$ is called the deformation (or, configuration) of the body $\mathcal{B}$ at time $t$. When $\chi$ is smooth enough we denote by :

$$
v(X, t)=\dot{x}=\frac{\partial \chi}{\partial t}(X, t) \text { and } \varepsilon(X, t)=\frac{\partial \chi}{\partial X}(X, t)-1>-1,
$$

the particle velocity and strain, respectively. We also denote by

$$
\varepsilon_{i}(X, t)=\frac{\partial^{i-1} \varepsilon(X, t)}{\partial X^{i-1}}=\frac{\partial^{i} \chi(X, t)}{\partial X^{i}}
$$

the $i$-th deformation gradient, $2 \leq i \leq r, r$ being a given positive integer and by

$$
\mathbf{g}(X, t)=\left(\varepsilon_{2}(X, t), \ldots, \varepsilon_{r}(X, t)\right),
$$

the $(r-1)$-tuple of the higher strain gradients.

For one dimensional bodies the equations of balance of momentum (in the absence of body forces) and mass can be written as follows:

$$
\rho \frac{\partial v}{\partial t}-\frac{\partial \sigma}{\partial X}=0, \quad \frac{\partial \varepsilon}{\partial t}-\frac{\partial v}{\partial X}=0 .
$$

where $\sigma=\sigma(X, t)$ is the nominal stress, i.e., the force per unit cross sectional area in the reference configuration. strain $\varepsilon$.

The system (4) must be completed by a constitutive equation relating the stress $\sigma$ and the

\subsection{Elastic approach}

The classical phenomenological description of phase transitions was generally made by using the constitutive model of thermo-elastic fluid suggested by van der Waals in 1873. By analogy the phenomenon of phase transformations in solid bars was modeled by certain authors by means of a non-monotone elastic constitutive equation. For example, Ericksen [6] and James [7] studied static solutions which contain stationary phase boundaries, while James [8] investigated the corresponding dynamic elastic bar theory, looking for solutions containing propagating phase boundaries. Attempts to describe martensitic phase transitions and shape memory effects by means of a Landau-Devonshire polynomial model has been made by Falk [9].

Thus, usually one supposes that the smooth stress-strain relation

$$
\sigma=\sigma_{R}(\varepsilon)
$$

has the property that $\sigma_{R}^{\prime}(\varepsilon)>0$ on $\left[0, \varepsilon_{\alpha}\right) \cup\left(\varepsilon_{\beta}, \infty\right)$ and $\sigma_{R}^{\prime}(\varepsilon)<0$ on $\left(\varepsilon_{\alpha}, \varepsilon_{\beta}\right)$ (Fig. 1a).

The elastic model (5) is compatible with the Clausius-Duhem inequality, which in the context of isothermal processes takes the form

$$
\rho \dot{\psi} \leq \sigma \dot{\varepsilon}
$$

where $\psi=\psi(X, t)$ is the free energy function at $(X, t)$ and the dot signifies the time derivative. It is known that for (5) there is a unique free energy function (modulo a constant), $\rho \psi_{R}(\varepsilon)=\int_{0}^{\varepsilon} \sigma_{R}(s) d s$, compatible with the second law of thermodynamics (6). Since (5) is a non-monotone stress-strain relation then the thermodynamic potential $\psi_{\Omega}(\varepsilon)$ is a non-convex one. Relying on this fundamental property, many efforts have been done to describe the hysteresis loops which occur during phase cransformations (see for instance [9]). But, this approach as a field theory has a major disadvantage. 
The system $(4+5)$ is of mixed type, hyperbolic-elliptic and the elastic problem is ill-posed in the sense that it may fail to have a solution in the phase transition interval $\left(\varepsilon_{\alpha}, \varepsilon_{\beta}\right)$ (see Suliciu [10]). On the other hand, the elastic system is not able to describe how the stress may deviate from the equilibrium.

\subsection{Rate-type viscoelastic approach}

One way to circumvent all these inconveniences has been proposed by Suliciu [11] (see also $[1,10,12$ 14]). The idea is to use a Maxwell's type viscoelastic model of the form

$$
\dot{\sigma}-E \dot{\varepsilon}=-k\left(\sigma-\sigma_{R}(\varepsilon)\right),
$$

where $E=$ const. $>0$ is dynamic Young's modulus, $1 / k=$ const. $>0$ is a relaxation time, and $\sigma_{R}(\varepsilon)$ is a non-monotone equilibrium stress-strain relation as in Fig. 1a.

In this case the system $(4+7)$ is always hyperbolic irrespective of the slope of the equilibrium curve $\sigma=\sigma_{R}(\varepsilon)$. Thus, the initial boundary value problems are theoretically well-posed. However, the system incorporates some physical instabilities due to the slope of the equilibrium curve (see [10]). The viscoelastic constitutive equation (7) generates a unique (modulo a constant) free energy function of strain and stress $\psi=\psi(\varepsilon, \sigma)$, compatible with the second law of thermodynamics $(6)$, which is also non-convex when the equilibrium curve $\sigma=\sigma_{R}(\varepsilon)$ is non-monotone (see $[10,12,13]$ and the references given there).

In a series of papers $[1,10-14]$, it was shown theoretically and numerically that this model may describe phenomenologically many aspects encountered in the transformation pseudoelasticity (shape memory alloys) such as the hysteretic behaviour in strain or stress controlled experiments, the nucleation and growth of one phase into another. Since the viscoelastic constitutive equation (7) can describe the way the states $(\varepsilon, \sigma)(X, t)$ may deviate from the equilibrium states $\left(\varepsilon, \sigma_{R}(\varepsilon)\right)(X, t)$, then the creation and propagation of phase boundaries is automatically accounted for this model. The results show that jerky flow and dynamic stress wave effects are involved during a stepped growth of strain bands.

This approach provides a reasonable tool for the investigation of the interplay between dissipation ("viscosity mechanism"), softening and inertia.

The two above approaches to phase transformations can be included in what is known in continuum mechanics as the theory of sharp phase transitions, in which the interface separating the two co-existent phases is viewed as a stationary or, propagating surface of discontinuity for the strain.

\subsection{Higher strain gradients approach}

Other modality, frequently used for the description of phase transformations is the theory of continuous phase transitions. In this case the interface between different phases of the material is treated as a thin transition layer, where the strain changes considerably, but smoothly.

Thus, in order to deal with phase boundaries of non-vanishing width it is assumed that the stress in equilibrium depends not only on the strain $\varepsilon$, but also on its higher strain gradients, i.e.,

$$
\sigma=\sigma_{R}(\varepsilon, \mathrm{g}), \quad \varepsilon>-1, \quad \mathrm{~g} \in R^{r-1},
$$

where $\mathrm{g}$ is the $(r-1)$-tuple of higher gradients of strain.

The gradient approach originates in the paper of van der Waals [15] from 1893 and has become a popular tool for the investigations of shear banding, localization of deformation $[3,4]$, phase transitions $[2,16-18]$ and other physical phenomena.

The most used constitutive relations of type (8) in literature is

$$
\sigma=\sigma_{R}(\varepsilon, \mathbf{g})=\sigma_{R}(\varepsilon)-a(\varepsilon) \varepsilon_{X X}-b(\varepsilon) \varepsilon_{X}^{2},
$$

where $a(\varepsilon)$ and $b(\varepsilon)$ are force-like coefficients, while $\sigma_{R}(\varepsilon)$ is a non-monotone stress-strain relation $[2,16-18]$; the subindice $X$ indicates partial derivative. Here, $\sigma_{R}(\varepsilon)$ denotes the usual homogeneous part of stress and the remaining part is an extra-stress induced by the microstructure of the medium and account for the effect of long-range molecular interactions. 
One-dimensional structured phase transitions under prescribed load, at equilibrium are obtained as solutions of the corresponding non-linear second-order differential equations $\sigma_{R}(\varepsilon, \mathbf{g})=$ $\sigma_{0}$, where $\sigma_{0}=$ const.. This problem was investigated for a finite interval by Falk [2] for its one-dimensional Ginzburg-Landau model for martensitic phase transitions in shape memory alloys (see also Carr et al. [18]) and for an infinite interval by Aifantis and Serrin [16,17] for fluid microstructures.

A main problem raised by the gradient approach is the compatibility of the constitutive structure with the second law. It is known that for the simplest constitutive models the dependence of the equilibrium response on the higher deformation gradients is incompatible with the thermodynamics based on the Clausius-Duhem inequality and the balance laws in their conventional form. For instance, it is easy to note that the second law in the form (6) rules out the dependence of the constitutive function (9) on the strain gradients.

There are two ways to overcome such too restrictive consequences of classical thermodynamics. The first one is based on the modification of the entropy inequality (more precisely of the entropy flux) (see Müller [19]) while the second one is based on the modification of the balance of energy (see Dunn and Serrin [20]) in an appropriate way (see also [21]).

l3oth approaches lead in a purely mechanical context to the following local form of the dissipation inequality:

$$
\rho \dot{\psi} \leq \sigma \dot{\varepsilon}+\mu_{X}
$$

where $\mu$ is a constitutive quantity as the free energy function $\psi$ and the stress $\sigma$. It may be called a (mechanical) flux of entropy (Müller [19]) or, an interstitial work flux (Dunn and Serrin [20]), due to the long range spatial interactions between the material elements, or particles of the body.

Now, the constitutive equation (8) is said to possess a free energy function compatible with the second law of thermodynamics in the form (10) if there exist a smooth function $\psi=\psi\left(\varepsilon, \varepsilon_{X}, \varepsilon_{X X}, \dot{\varepsilon}\right)$ and an energy (or, entropy flux) $\mu=\mu\left(\varepsilon, \varepsilon_{X}, \varepsilon_{X X}, \dot{\varepsilon}\right)$ such that inequality (10) be satisfied for any motion $x=\chi(X, t)$ of the body.

By using a standard procedure one gets that a free energy function for equation (9) exists if and only if

$$
b(\varepsilon)=\frac{1}{2} a^{\prime}(\varepsilon) .
$$

The general form of the free energy function $\psi$ and of the flux $\mu$ are then

$$
\rho \psi=\rho \psi\left(\varepsilon, \varepsilon_{X}\right)=\frac{1}{2} a(\varepsilon) \varepsilon_{X}^{2}+m(\varepsilon) \varepsilon_{X}+\psi_{R}(\varepsilon), \quad \mu=\mu\left(\varepsilon, \varepsilon_{X}, \dot{\varepsilon}\right)=\left(a(\varepsilon) \varepsilon_{X}+m(\varepsilon)\right) \dot{\varepsilon}
$$

where $\psi_{R}(\varepsilon)=\int_{0}^{\varepsilon} \sigma_{R}(s) d s$ and $m(\varepsilon)$ is an arbitrary function.

In this case there is no dissipation and the inequality $(10)$ is satisfied with the sign equal. For $m(\varepsilon)=0$ the free energy function (12) is just the free energy proposed by van der Waals [15] and used by Falk [2]. On the other hand, condition (11) implies that the equilibrium solutions corresponding to the model (9) satisfy Maxwell's rule (like the classical theories) (see Aifantis and Serrin [16,17]). If $a(\varepsilon)=a=$ const. we get from (11) that $b(\varepsilon)=0$. This case has been considered by Falk [2] for the one-dimensional static Ginzburg-Landau theory for the martensitic phase transitions in shape memory alloys and by Sprekels and Zheng [22] for the study of smooth solutions of the system of partial differential equations governing the dynamics of martensitic phase transitions.

Although the modification of the dissipation inequality partially clarify the thermodynamical aspects concerning non-simple materials, the delicate question: which is the appropriate thermodynamical structure for this class of materials is still open (see also [21]). Indeed, Šilhavý [23] pointed out that materials with internal variables can have non-simple equilibrium response within the framework of the conventional thermodynamics based on the unmodified Clausius-Duhem inequality and balance laws. Such an example is given in the next section.

\subsection{A rate-type gradient approach}

Starting from the good results obtained in modelling transformation pseudo-elasticity as well as plastic flow localization by means of Maxwell's rate-type constitutive equation (7) (see [1, 10-14]) anc' l,aking into account that certain phenomena can be described within the framework of continuum mechanics only if the stress in equilibrium depends on the higher gradients of deformation 
we have proposed a new model [5] which combines the rate-type viscoelastic approach (7) and the gradient approach (9).

Since the strain gradients may be thought as a manifestation of the various microprocesses that may occur at the microscale, our aim was to allow for the possibility of obtaining spatial patterns of the microstructures.

We supposed that our one-dimensional bar obeys the non-local rate-type constitutive equation:

$$
\dot{\sigma}-E \dot{\varepsilon}=G(\varepsilon, \sigma, \mathbf{g}),
$$

where $E=$ const. $>0$ and $G:(-1, \infty) \times R \times R^{r-1} \rightarrow R$ is a relaxation function which depends on the first $r$ strain gradients.

Let us denote the kernel of the constitutive function $G(\varepsilon, \sigma, g)$ by

$$
\mathcal{E}=\left\{(\varepsilon, \sigma, \mathbf{g}) \mid G(\varepsilon, \sigma, \mathbf{g})=0, \quad(\varepsilon, \sigma, \mathbf{g}) \in(-1, \infty) \times R \times R^{r-1}\right\} .
$$

The set $\mathcal{E}$ is called the elastic set, or the equilibrium set of the constitutive equation. Therefore, a state $S=(\chi(X), \sigma(X))$ is said to be an elastic state (or, an equilibrium state) of the body $\mathcal{B}$ if $G(\varepsilon(X), \sigma(X), \mathrm{g}(X))=0$, for any $X \in \mathcal{R}$.

We denote by

$$
\varepsilon^{p}=\varepsilon-\frac{\sigma}{E} \quad \text { and } \quad \varepsilon^{e}=\frac{\sigma}{E}
$$

the inelastic strain and the elastic strain at the state $S=(\chi, \sigma)$, respectively. Relation (14) implies that the inelastic strain of the body $\mathcal{B}$ at an equilibrium state is permanent.

We add now to relation (14) two essential constitutive assumptions. First, we suppose that

$$
G(\varepsilon, \sigma, 0)=0 \text { if and only if } \sigma=\sigma_{R}(\varepsilon),
$$

where $\sigma_{R}:\left(\varepsilon_{I}, \varepsilon_{I I}\right) \rightarrow R, \quad \sigma_{R}(0)=0, \quad \sigma_{R} \in C^{0}\left(\varepsilon_{I}, \varepsilon_{I I}\right)$ is called the homogeneous equilibrium curve. That is, the homogeneous equilibrium set reduces to a unique curve in the $\varepsilon-\sigma$ space.

Second, we suppose that

$$
\left(\sigma-\sigma_{R}(\varepsilon)\right) G(\varepsilon, \sigma, 0) \leq 0, \quad \text { for any }(\varepsilon, \sigma) \in\left(\varepsilon_{I}, \varepsilon_{I I}\right) \times R, .
$$

This assumption represents the necessary and sufficient condition for the homogeneous equilibrium curve $\sigma=\sigma_{R}(\varepsilon)$ to be stable with respect to homogeneous relaxation processes.

It is obvious that our constitutive model coincides on the set of homogeneous state (i.e., when $\mathrm{g} \equiv 0)$ to the viscoelastic rate-type Maxwell's model (7). A main advantage of the present approach relies on the fact that its constitutive structure, although depending on higher strain gradients, is compatible with the classical form of the dissipation inequality $(6)$.

A smooth process of the body $\mathcal{B}$ will be a pair $(\chi(\cdot, t), \sigma(\cdot, t)), t \in\left[0, T_{0}\right)$ of smooth time dependent fields over $\mathcal{R}$ which satisfies the constitutive equation (14).

We say that the constitutive equation (14) generates a free energy function of strain, stress and higher strain gradients, compatible with the second law of thermodynamics if there exists a smooth function $\psi=\psi(\varepsilon, \sigma, \mathrm{g}), \psi:\left(\varepsilon_{I}, \varepsilon_{I I}\right) \times R \times R^{r-1} \rightarrow R$, such that the dissipation inequality (6) he satisfied for any process $(\chi(\cdot, t), \sigma(\cdot, t)), t \in\left[0, T_{0}\right)$.

In [5] we have shown that the above requirement is equivalent to the following conditions:

i) the free energy is independent of $\mathrm{g}$, i.e., $\psi=\psi(\varepsilon, \sigma)$

ii) the following relations

$$
\frac{\partial \psi}{\partial \varepsilon}(\varepsilon, \sigma)+E \frac{\partial \psi}{\partial \sigma}(\varepsilon, \sigma)=\frac{1}{\rho} \sigma, \quad \frac{\partial \psi}{\partial \sigma}(\varepsilon, \sigma) G(\varepsilon, \sigma, \mathbf{g}) \leq 0
$$

hold for every $(\varepsilon, \sigma, \mathbf{g}) \in\left(\varepsilon_{I}, \varepsilon_{I I}\right) \times R \times R^{r-1}$.

Moreover, we showed that the constitutive relation (14), satisfying assumptions $(17+18)$, has a unique free energy function (modulo a constant) if and only if

$$
\frac{\sigma_{\Omega}\left(\varepsilon_{1}\right)-\sigma_{R}\left(\varepsilon_{2}\right)}{\varepsilon_{1}-\varepsilon_{2}}<E, \quad \text { for any } \varepsilon_{1}, \varepsilon_{2} \in\left(\varepsilon_{1}, \varepsilon_{I I}\right), \varepsilon_{1} \neq \varepsilon_{2}
$$


and

$$
\left(\sigma-\sigma_{R}(\varepsilon)\right) G(\varepsilon, \sigma, \mathrm{g}) \leq 0, \quad \text { for any }(\varepsilon, \sigma, \mathrm{g}) \in\left(\varepsilon_{I}, \varepsilon_{I I}\right) \times R \times R^{r-1} .
$$

More than that, the free energy function of our non-local model (14) is just the free energy function of the local Maxwell's type viscoelastic model (7) (see [10-12] and the references therein).

Thus, the second law of thermodynamics imposes explicit necessary and sufficient restrictions on the constitutive functions $G(\varepsilon, \sigma, \mathbf{g}), \sigma_{R}(\varepsilon)$ and $E$ of our model (14).

The main problem is now to investigate how the constitutive restriction (21) limits the dependence of function $G$ on the higher strain gradients and to give a non-trivial example.

We must note here that our non-local rate-type constitutive equation (14) may be viewed as a constitutive model for a non-simple body with one internal state variable. Indeed, according to relations (16) we may write (14) under the equivalent form

$$
\sigma=\hat{\sigma}\left(\varepsilon, \mathbf{g}, \varepsilon^{p}\right)=E\left(\varepsilon-\varepsilon^{p}\right), \quad \dot{\varepsilon}^{p}=\hat{G}\left(\varepsilon, \mathbf{g}, \varepsilon^{p}\right)=-\frac{1}{E} G\left(\varepsilon, E\left(\varepsilon-\varepsilon^{p}\right), \mathbf{g}\right),
$$

where $\hat{\sigma}$ and $\hat{G}$ are two constitutive functions for a body of grade $\mathrm{r}$ with one internal state variable.

Examples of one-dimensional plasticity theories with internal state variables in which the inelastic strain $\varepsilon^{p}$ may be also interpreted as an internal state variable can be found in the papers by Kratochvil \& Dillon (see for instance [24]).

Thus, our non-local rate-type constitutive equation (14) may be considered as a specific case of the theory of bodies of grade $r$ with internal variables studied by Silhavý in [23]. The main difference between our approach and that of Šilhavý relies in fact on the manner to define the equilibrium set (15) of the constitutive model. Since Šilhavý deals exclusively with equilibrium situation it was not necessary to characterize the behaviour of the processes of the body outside the equilibrium set. Therefore, he does not obtain explicit restrictions of the type (20-21) on the constitutive functions which describe the evolution of the internal state variables. Unlike this approach we are more interested in describing the dynamics of phase transitions. That is why we have introduced assumption (18) concerning the stability of homogeneous relaxation processes with respect to the equilibrium curve. Thus, this condition as well as its thermodynamical consequence (21) must be understood as a characterization of the way the processes of the body may deviate from equilibrium states without contradicting the second law.

In order to define the equilibrium set of the constitutive structure Šilhavý used throughout his papers the following assumption which seems to be inadequate in this context. He supposed that, for each $(\varepsilon, \mathrm{g}) \in\left(\varepsilon_{I}, \varepsilon_{I I}\right) \times R^{r-1}$ there is exactly one value $\sigma=\sigma_{R}(\varepsilon, \mathrm{g})$ such that

$$
G\left(\varepsilon, \sigma_{R}(\varepsilon, \mathbf{g}), \mathbf{g}\right)=0, \quad \text { for any }(\varepsilon, \mathbf{g}) \in\left(\varepsilon_{I}, \varepsilon_{I I}\right) \times R^{r-1}
$$

In other words, the equilibrium set (15) is supposed to be a hypersurface $\sigma=\sigma_{R}(\varepsilon, \mathrm{g})$ in the space $(\varepsilon, \sigma, g)$. It is easy to see now that such a definition of the equilibrium set coupled with the thermodynamical restriction (21) will lead to an incompatibility with the second law, or will remove the dependence of the equilibrium response function $\sigma=\sigma_{R}(\varepsilon, \mathbf{g})$ on the set of higher strain gradients $\mathrm{g}$.

In conclusion, the equilibrium set $\mathcal{E}$ of the non-local model can not be a hypersurface in the $(\varepsilon, \sigma, \mathrm{g})$ space, but a closure of an open set. We remind the reader that a local constitutive equation of the form (14) is called viscoplastic if the set where $G(\varepsilon, \sigma)=0$ is a suitable region in the $\varepsilon-\sigma$ space and it is called viscoelastic if that set is a single curve [25]. Therefore, a non-local rate-type constitutive equations (14) compatible with the conventional thermodynamics based on the unmodified Clausius-Duhem inequality describe always a viscoplastic behaviour.

We give now a simple and useful example of a non-local rate-type viscoplastic constitutive equation (14), compatible with the second law of thermodynamics.

We suppose that the relaxation function $G$ is given by

$$
G(\varepsilon, \sigma, \mathbf{g})= \begin{cases}-k\left(\sigma-\sigma_{R}(\varepsilon)-\kappa(\mathbf{g})\right), & \text { for } \sigma-\sigma_{R}(\varepsilon)>\kappa(\mathbf{g}), \\ 0, & \text { for }\left|\sigma-\sigma_{R}(\varepsilon)\right| \leq \kappa(\mathbf{g}), \\ -k\left(\sigma-\sigma_{R}(\varepsilon)+\kappa(\mathbf{g})\right), & \text { for } \sigma-\sigma_{R}(\varepsilon)<-\kappa(\mathbf{g}),\end{cases}
$$

where $k=$ const. $>0$ is a viscosity coefficient. Function $\sigma=\sigma_{R}(\varepsilon)$, which characterizes the homogeneous equilibrium states is a non-monotone one (Fig. 1a) and it is supposed to satisfy the 
thermodynamic restriction (20). Function $\kappa=\kappa(\mathrm{g}), \kappa: R^{r-1} \rightarrow R$, which is responsible for the inhomogeneities induced by the microstructures of the material is a positive one, i.e., $\kappa=\kappa(\mathrm{g})>0$, for any $\mathbf{g} \in R^{r-1}, \mathbf{g} \neq 0$, and $\kappa(\mathbf{0})=0$.

It is easy to verify now that when $\kappa(\mathrm{g}) \equiv 0$ our gradient-dependent viscoplastic model $(14+24)$ reduces to the local Maxwell's rate-type viscoelastic constitutive equation (7).

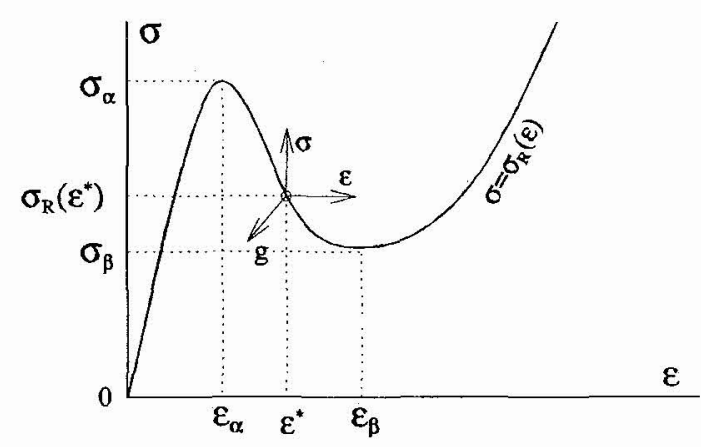

a)

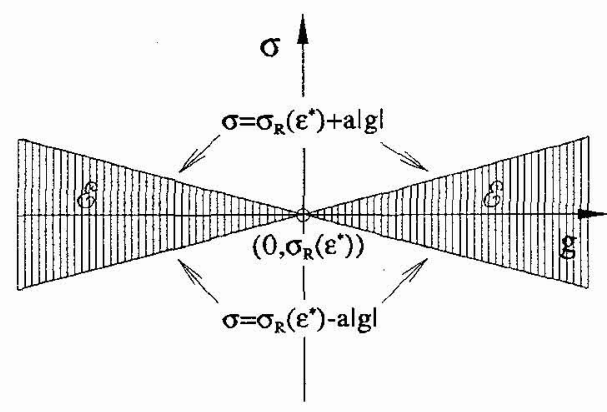

b)

Figure 1: a) The homogeneous equilibrium curve.

b) Section through the equilibrium set $\mathcal{E}$ for an $\varepsilon=\varepsilon^{*}$.

In the numerical experiments from the next section we take $\sigma=\sigma_{R}(\varepsilon)$ a piecewise, linear non-monotone stress-strain relation, i.e.,

$$
\sigma_{R}(\varepsilon)=\left\{\begin{array}{lll}
E_{1} \varepsilon, & \text { if } \quad 0 \leq \varepsilon \leq \varepsilon_{\alpha}, \\
-E_{2}\left(\varepsilon-\varepsilon_{\alpha}\right)+\sigma_{\alpha}, & \text { if } \quad \varepsilon_{\alpha}<\varepsilon<\varepsilon_{\beta}, \\
E_{3}\left(\varepsilon-\varepsilon_{\beta}\right)+\sigma_{\beta}, & \text { if } \quad \varepsilon_{\beta} \leq \varepsilon
\end{array}\right.
$$

and the extra-stress function $\kappa=\kappa(\mathrm{g})$ is taken as

$$
\kappa(\mathrm{g})=a\left|\varepsilon_{X X}\right|
$$

where $a=$ const. $>0$ is a scale factor. The constants $E_{1}, E_{2}, E_{3}, \sigma_{\beta}, \sigma_{\alpha}, \varepsilon_{\beta}, \varepsilon_{\alpha}$ are subjected to the following restrictions, which are in agreement with (20) $0<E_{1}<E, 0<E_{3}<E, E_{2}>0$, $\sigma_{\alpha}>\sigma_{\beta}>0,0<\varepsilon_{\alpha}<\varepsilon_{\beta}, \sigma_{\alpha}-\sigma_{\beta}=E_{2}\left(\varepsilon_{\beta}-\varepsilon_{\alpha}\right)$.

The equilibrium, or the elastic set is in this case the domain

$$
\mathcal{E}=\left\{(\varepsilon, \sigma, g)|| \sigma-\sigma_{R}(\varepsilon)|\leq a| g \mid, \sigma \geq 0, \varepsilon \geq 0, g=\varepsilon_{X X}\right\} \subset R^{+} \times R^{+} \times R .
$$

bounded by the hypersurfaces $\sigma=\sigma_{R}(\varepsilon) \pm a \varepsilon_{X X}$ in the $(\varepsilon, \sigma, \mathbf{g})$ space (see Fig. 1b)

The influence of the rate-dependent effects ("viscous mechanism"), the influence of the slope of the homogeneous equilibrium curve $\sigma=\sigma_{R}(\varepsilon)$ ("softening mechanism"), and of the internal length scale ("strain gradients effects") on the way the instabilities may develop in the dynamic process of phase transformations are investigated in [5]. In order to do that we study the stability/instability of an homogeneous equilibrium state with respect to a small perturbation. This analysis is similar in certain respect to the familiar linear stability analysis. All the above three mechanisms compete in the case of our viscoplastic model $(14+24)$ at the localization and propagation of deformation.

\section{NUMERICAL RESULTS}

We consider in the following the system of partial differential equations $(4+14)$, where $G, \sigma_{R}(\varepsilon)$ and $\kappa(\mathrm{g})$ are given by $(24),(25)$ and (26), respectively. It is known that the above system is hyperbolic when the relaxation modulus $G$ is independent on the higher deformation gradients, for instance when $G=0$, and it is of parabolic type at the points $(X, t) \in[0, L] \times\left[0, T_{0}\right)$ where $G$ 
depends on $g$. In this case we have to supplement the boundary data by appropriate conditions for the strain gradients.

In the numerical experiments presented here we consider a bar in its natural state subjected to a constant strain rate test. Thus we have to solve the following initial-boundary value problem:

$$
\begin{aligned}
& \varepsilon(X, 0)=0, \quad \sigma(X, 0)=0, \quad v(X, 0)=0, \quad \text { for any } X \in[0, L], \\
& v(0, t)=v^{*}=\text { const., } \quad v(L, t)=0, \quad \text { for any } t \geq 0, \\
& \varepsilon_{X}(0, t)=0, \quad \text { for any } t>0 \text { for which } G(\varepsilon, \sigma, g)(0, t) \neq 0, \\
& \varepsilon_{X}(L, t)=0, \quad \text { for any } t>0 \text { for which } G(\varepsilon, \sigma, g)(L, t) \neq 0,
\end{aligned}
$$

In order to solve numerically the above dynamic problem we developed a finite difference scheme which reduces for $a=0$, i.e., when the the model (14) becomes (7) and the system $(4+14)$ is of hyperbolic type, to the numerical method of characteristics as described in [26]. The numerical scheme verifies a numerical stability condition on the time integration step which ensures that the total (numerical) energy of an isolated body problem (i.e., (28) with $v^{*}=0$ ) is non-increasing in time. This condition is a consequence of the second law, more precisely on the fact that the numerical solution satisfies the dissipation inequality (21). As a result we get that the first approximation of the numerical method is stable when the time integration step verifies the inequality $\tau \leq \frac{2}{k} \frac{E-\max \left(E_{1}, E_{3}\right)}{E+E_{2}}$ which is in fact the same restriction on the time integration step as that obtained in [26]. A systematic numerical study will be presented elsewhere.

In the numerical experiments performed in this paper we have used the following entries: $E=2250 \mathrm{kgf} / \mathrm{mm}^{2}, E_{1}=E_{3}=2200 \mathrm{kgf} / \mathrm{mm}^{2}, E_{2}=566,6 \mathrm{~kg} / \mathrm{mm} \mathrm{m}^{2}, \rho=7500 \mathrm{~kg} / \mathrm{m}^{3}, \varepsilon_{\alpha}=0.01$, $\varepsilon_{\beta}=0.04, \sigma_{\alpha}=22 \mathrm{kgf} / \mathrm{mm}^{2}, \sigma_{\beta}=5 \mathrm{kgf} / \mathrm{mm}^{2}$. These values are appropriate for a shape memory alloy (see for example Otsuka et al. [27]). The specimen length is $L=20 \mathrm{~mm}$ and the end velocity of the bar $v^{*}=-1 \mathrm{~mm} / \mathrm{sec}$, which corresponds to the imposed engineering strain rate $\dot{\varepsilon}_{e}=0.05 \mathrm{sec}^{-1}$. We take for the scale factor a value close to that proposed by Zbib and Aifantis [4] for a steel specimen i.e., $a=0.5 \mathrm{kgf}$, while for the viscosity coefficient we take the value $k=5 \times 10^{-5} \mathrm{sec}^{-1}$. We used a fixed mesh with 201 points. That means that the maximal length of a strain band which can be accounted is of $0.1 \mathrm{~mm}$.

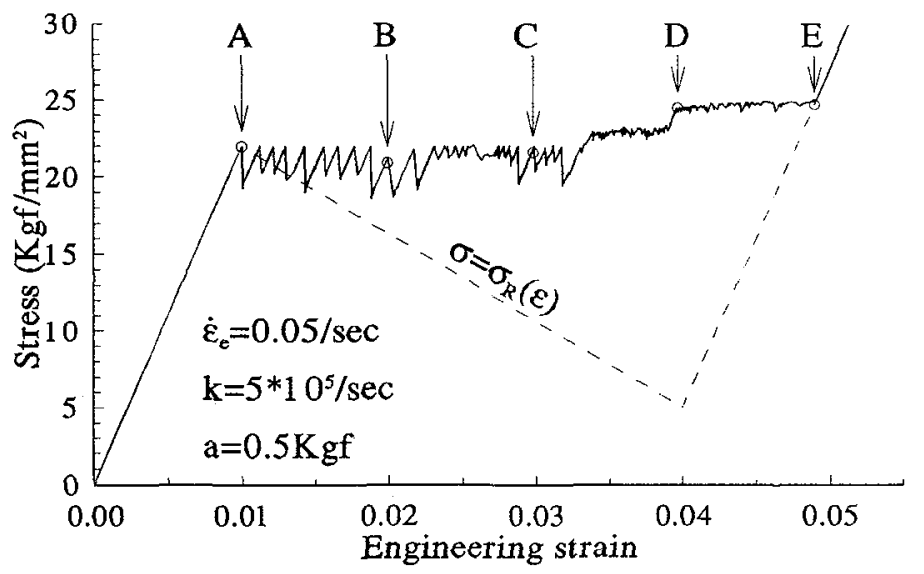

Figure 2: End stress $\sigma(0, \mathrm{t})$ versus engineering strain in a strain controlled experiment.

The evolution in time of the stress, strain and particle velocity fields over the entire specimen is then calculated as solution of the initial-boundary value problem (28) for the system $(4+14)$, without disregarding the inertial term in the field equations.

In Fig 2. we plot the end stress $\sigma(0, t)$ versus the engineering strain $\varepsilon_{e}(t)=\frac{1}{L} \int_{0}^{L} \varepsilon(t, X) d X$. The point $A$ corresponds to the moment where the initially austenitic sample starts changing phase, i.e., where the spatial homogeneity of strain and stress fields is lost. The point E corresponds to the moment where this homogeneity is recovered and the specimen is entirely in the martensitic 
phase. The strain profiles in Figs. 3 a)-c) are similar to the regular patterns of martensitic and austenitic stripes (fine structures) appearing during stress-induced phase transformations of shape memory single crystals (see for instance Otsuka et al.[27]).

Indeed it is known that hysteresis is accompanied by a domain structure of coexisting martensitic and austenitic domains. In Figs. 3 a)-c) one can see how martensitic domains grow at the expense of the austenitic domains and how the transformation proceeds by domain boundary movement. Due to the presence of higher strain gradients in the constitutive equation there are no sharp interfaces between the different phases of the material.

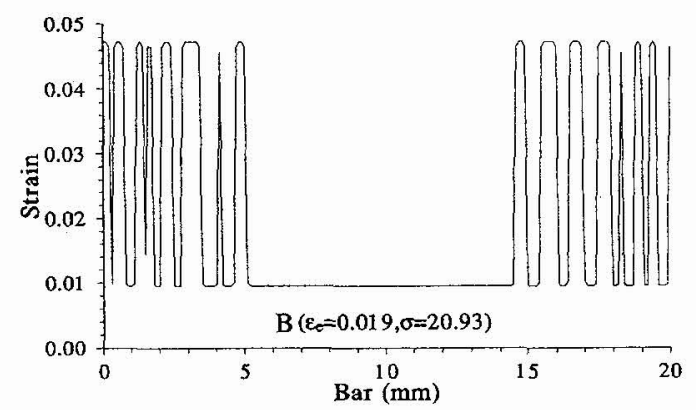

a)

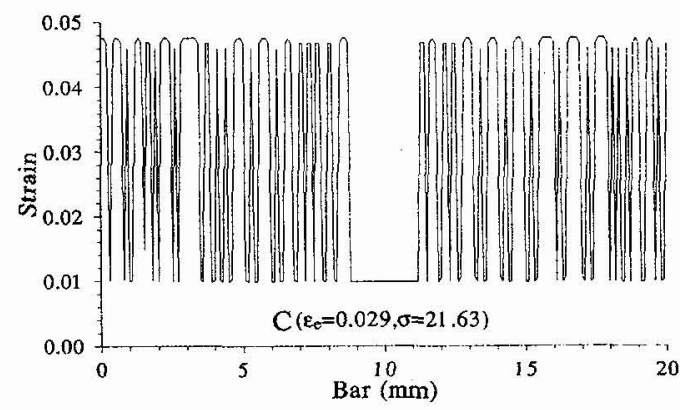

b)

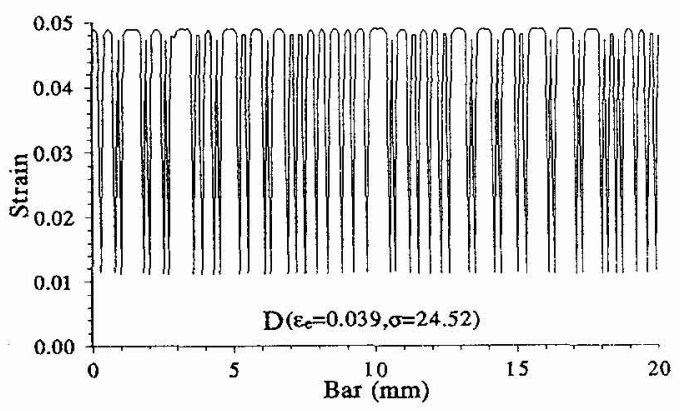

c)

Figure 3: Evolution of layered structures. Local strain $\varepsilon(X, t)$ versus bar at three moments marked on Fig. 2.

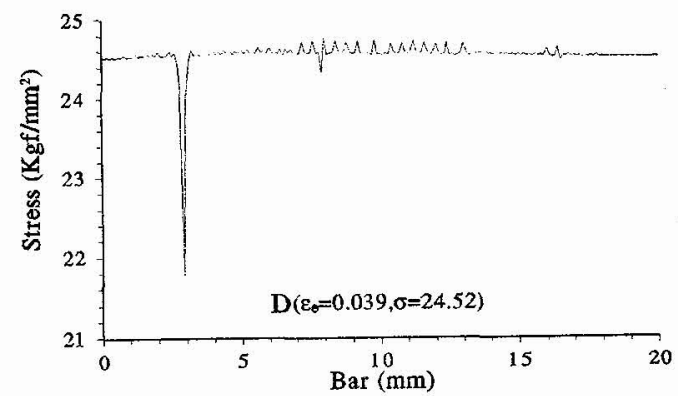

a)

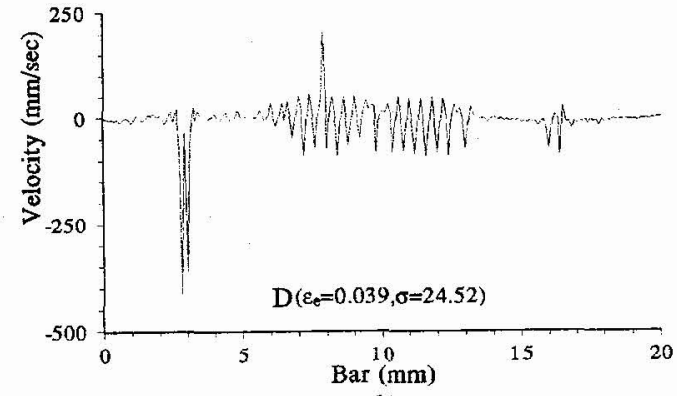

b)

Figure 4: Distribution of stress and particle velocity in the bar at the moment D on Fig. 2

The solution preserves many characteristics of the strain controlled processes in rate-type viscoelastic bars of type (7) (see $[1,13,14]$ for comparison). For example, in the local model as well as in non-local one, one can observe a drop in load at the yield point and a sawtooth curve, in which the stress alternately rises and fails with abrupt changes of slope at the points of sawteeth. A difference appears on the last part of the stress-strain curve, where the amplitude of the serrations 
decreases while the stress increases exceeding significantly the value of $\sigma_{\alpha}$ before the transformation be complete.

In Figs. 4 a)-b) we have ploted the distribution of stress and particle velocity along the specimen at a moment where dynamic effects and instabilities phenomena are involved. They illustrate that even if the imposed engineering strain rate is small it is unreasonable to neglect the inertial term in the balance equation (4).

\section{Acknowledgements}

This work has been started while C. F. was holding a visiting position at the Université de Metz, Laboratoire Physique et Mécanique des Matériaux, URA CNRS No. 1215 and continued while he benefiting of a fellowship from the Alexander von Humboldt Foundation at Technische Universität Berlin. The support and the hospitality of the above institutions are gratefully acknowledged.

\section{References}

[1] Făciu C. and I. Suliciu, Scripta Metall. 31 (1994) 1399-1404.

2] Falk F., Z. Physik B - Condensed Matter 51 (1983) 177-185.

3) Coleman B.D. and Newman D.C., Acta Mech. 78 (1989) 263-279.

4) Zbib H.M.and Aifantis E.C., Acta Mech. 92 (1992) 209-225.

[5] Făciu C. and Molinari A., A non-local rate-type viscoplastic approach to patterning of deformation. (to be published).

6] Ericksen J.L, J. Elasticity 5 (1975) 191-201.

7 James R.D., Arch. Rat. Mech. Anal. 72 (1979) 99-140.

8) James R.D., Arch. Rat. Mech. Anal. 73 (1980) 125-158.

9] Falk F., Acta Metall. 28 (1980) 1773-1780.

[10] Suliciu I., Int. J. Engng. Sci. 30 (1992) 483-494.

11] Suliciu I., On the description of the dynamics of phase transitions by means of rate-type constitutive equations. A model problem. In Plasticity '89, Eds. A. S. Khan and M. Tokuda, (Pergamon Press, Oxford, 1989) pp. 417-420.

12) Făciu C., Int. J. Engng. Sci. 29 (1991) 1103-1119.

13) Făciu C., J. Phys. IV France, Colloque C4, 1 (1991) 101-106.

14 Mihăilescu-Suliciu M. and Suliciu I., Mech. Res. Comm. 20 (1993) 475-480.

[15] van der Waals J.D., Verhandel. Konink. Akad. Weten. Amsterdam (Sect. 1) 1 (1893), English translation Rowlinson, J.S. J. Stat. Phys. 20 (1979) 197-244.

[16] Aifantis E.C. and Serrin J.B., J. Colloid Iterface Sci. 96 (1983) 530-547.

17. Aifantis E.C. and Serrin J.B., J. Colloid Iterface Sci. 96 (1983) 517-529.

18. Carr J., Gurtin M.E. and Slemrod M., J. Elasticity 15 (1985) 133-142.

19 Müller I., Arch. Ration. Mech. Anal. 26 (1967) 118-141.

20] Dunn J.E. and Serrin J., Arch. Ration. Mech. Anal. 88 (1985) 95-133.

21] Aifantis E.C., Mech. Res. Comm. 5 (1978) 139-145.

22] Sprekels J. and Zheng S., Physica D 39 (1989) 59-76.

[23] Šilhavý M., Arch. Ration. Mech. Anal. 88 (1985) 135-161.

24) Kratochvil J. and Dillon O.W., J. Appl. Phys. 41 (1970) 1470-1479.

125 Suliciu I. and Sabac M., Math. Anal. Appl. 131 (1988) 354-372.

26. Mihăilescu-Suliciu M. and Suliciu I., ZAMM 72 (1992) 667-674.

27. Otsuka K., Wayman C.M., Nakai K., Sakamoto H., Shimizu K., Acta Metall. 24 (1976) $207-226$. 